Revista de Comunicación y Salud, 2018, Vol. 8, n² 2, pp. 41-49

Editado por Cátedra de Comunicación y Salud

ISSN: 2173-1675

Enviado 26 de septiembre de 2018

Aprobado 28 de octubre de 2018

\title{
"RUIDO" EN EL PROCESO DE COMUNICACIÓN EN LOS PROSPECTOS DE LOS MEDICAMENTOS, COMO PARTE DE LA LITERATURA GRIS
}

"Noise" in the process of communication in the prospectus of medicines as a part of the Gray Literature

Ángel López Gutiérrez ${ }^{1}$

Profesor de Idiomas en ESIC. Pozuelo de Alarcón. Madrid.

María Julia Bordonado Bermejo

Doctora en Ciencias Económicas y Empresariales y Licenciada en Derecho. Profesora de Derecho de la Comunicación e Historia Económica en ESIC, (URJC)

\section{Resumen}

Introducción: los prospectos incluidos en los envases de los medicamentos, son documentos informativos que forman parte de la "Literatura Gris". La información escrita que aparece en los prospectos de todos los medicamentos se realiza mediante el uso de un lenguaje muy técnico y específico que no todos pueden entender fácilmente. Objetivo: identificar y analizar los problemas de comprensión que muchos de los potenciales usuarios podemos sufrir al leer la información correspondiente al prospecto de una especialidad medicinal de venta libre, o con receta médica, debido al uso de términos específicos que no son conocidos por una gran parte de la población. Método: se basa en la realización de una encuesta dirigida a un público objetivo entre los 18 y los 65 años o más, y de ambos sexos. La encuesta se realizó a través de internet durante los meses de Julio y parte de Agosto de 2018 en todo el territorio español. Resultados: se estableció un diagnostico de la situación, se crearon una serie de preguntas sobre los hábitos de lectura de los prospectos por los distintos grupos de edad de la población española, muchos participantes comunicaron sus propias ideas sobre el cambio necesario en el lenguaje utilizado en los prospectos de las medicinas y recalcaron la necesidad de una mayor implicación en los procesos de comunicación por parte del médico y del farmacéutico. Conclusiones: basadas en los resultados de las encuestas. Según las mismas, se harán propuestas a la Autoridad competente para mejorar la comunicación de los prospectos sanitarios destinados al consumo y uso de la población. Por ejemplo, utilización de comunicación visual en los prospectos y sinónimos de las palabras técnicas, según su etimología. Como puede ser, gastritis: inflamación del estómago.

Palabras clave: comunicación, información, "ruido", prospectos, medicamentos.

\footnotetext{
${ }^{1}$ Autor para correspondencia: Ángel López Gutiérrez angel.lopez@esic.edu
} 
"Ruido" en el proceso de comunicación en los prospectos de los medicamentos, como parte de la Literatura Gris.

\begin{abstract}
Introduction: the leaflets included in the packaging of the medicines are informative documents that are part of the "Gray Literature". The written information that appears on the leaflets of all medicines is made through the use of a very technical and specific language that not everyone can easily understand. Objective: to identify and analyze the comprehension problems that many of the potential users may suffer when reading the information corresponding to the prospectus of an over-the-counter medicinal specialty, or with a prescription, due to the use of specific terms that are not known by a large part of the population. Method: it is based on conducting a survey aimed at a target audience between 18 and 65 years of age or older, and of both sexes. The survey was conducted through the Internet during the months of July and a part in August of 2018 throughout the Spanish territory. Results: a diagnosis of the situation was established, a series of questions were created on the reading habits of the prospectus by the different age groups of the Spanish population, many participants communicated their own ideas about the necessary change in the language used in the medicine prospectus and stressed the need for greater involvement in communication processes by the doctor and the pharmacist. Conclusions: based on the results of the surveys. According to them, proposals will be made to the competent authority to improve the communication of the health leaflets destined to the consumption and use of the population. For example, use of visual communication in prospectus and synonyms of technical words, according to their etymology. For instance, gastritis: inflammation of the stomach.
\end{abstract}

Keywords: communication, information, "noise", prospects, medicines.

\title{
Cómo citar el artículo
}

López Gutiérrez, A. y Bordonado Bermejo, M. J. (2018). "Ruido" en el proceso de comunicación en los prospectos de los medicamentos, como parte de la Literatura Gris. Revista de Comunicación y Salud, 8(2), 41-49.

doi: http://doi.org/10.35669/revistadecomunicacionysalud.2018.8(2).41-49

\section{INTRODUCCIÓN}

"Entiendo la comunicación como el acto de relación entre dos o más sujetos, mediante el cual se evoca en común un significado". (Paoli, 1983, p. 11).

La utilización de prospectos para transmitir información importante para los consumidores voluntarios e involuntarios de los medicamentos, entra dentro de la llamada "Literatura Gris" que fue definida en la cuarta Conferencia Internacional de Literatura Gris celebrada en Washington DC en octubre de 1999.

La "Literatura Gris" es "todo lo que se produce en todos los niveles de gobierno, académicos, empresas y la industria en forma impresa y en formato electrónico, pero que no está controlado por los editores comerciales". La Universidad de Nueva Inglaterra (une), lo define: "The term grey literature refers to research that is either unpublished or has been published in non-commercial form".

Los prospectos incluidos en los envases de los medicamentos, son documentos informativos que forman parte de la "Literatura Gris". La información escrita que

Revista de Comunicación y Salud, 2018, Vol. 8, nº 2, pp. 41-49 
"Ruido" en el proceso de comunicación en los prospectos de los medicamentos, como parte de la Literatura Gris.

aparece en los prospectos de todos los medicamentos se realiza mediante el uso de un lenguaje muy técnico y específico que no todos pueden entender fácilmente.

En el caso de la medicina, el uso de una jerga específica se ha convertido en norma. La mayoría de la población apenas conoce los términos más comunes, por lo que es difícil de entender el prospecto con la información del medicamento que ha sido prescrito por el médico.

El término comunicación procede de la palabra latina communicare cuyo significado es "hacer partícipe a otro de lo que uno tiene". La Comunicación es, pues, un proceso en el que se intercambia información. Un emisor transmite a un receptor una información a través de un canal, esperando una respuesta del receptor y siempre dentro de un contexto determinado.

El elemento perturbador para una buena comunicación, aparece en el canal y se llama ruido. El ruido es cualquier interferencia que se produzca en la comunicación y que pueda impedir que esta se realice con éxito. Para que exista una buena comunicación han de concurrir dos condiciones, que el canal funcione adecuadamente y que no exista ruido. Además, también tiene que ocurrir que el receptor conozca el código en el que está cifrado el mensaje, si se desconoce el código que está utilizando el emisor, el mensaje no tendrá significado para el receptor y la comunicación será imposible. Esto ocurre, en un gran número de ocasiones, con los prospectos de los medicamentos.

En el caso de los prospectos, el tipo de comunicación que se establece es escrita. La definición de prospecto se encuentra regulada en el art. 8.1 del Real Decreto Artículo 8.1. del Real Decreto 2236/1993, de 17 de diciembre, por el que se regula el etiquetado y prospecto de los medicamentos de uso humano.

El prospecto es la información escrita que acompaña al medicamento, dirigida al consumidor o usuario. Mediante él se identifica al titular de la autorización y, en su caso, al fabricante, se menciona su composición y se dan instrucciones para su administración, empleo y conservación, así como sus efectos adversos, interacciones, contraindicaciones y demás datos que se determinan en el anexo III de este Real Decreto, con el fin de proponer su más correcto uso y la observancia del tratamiento prescrito, así como las medidas a adoptar en caso de intoxicación.

Todos los prospectos tienen los mismos apartados para facilitar la búsqueda de la información al usuario: composición, indicaciones, posología, contraindicaciones, precauciones, efectos secundarios e interacciones. En la redacción de los textos de los prospectos de los medicamentos las frases farragosas, los tecnicismos y los términos dudosos son muy habituales. El lenguaje técnico-científico puede ser muy difícil de entender.

Los aspectos legales que regulan los prospectos vienen señalados en la web de la Agencia Española del Medicamento (AEM), y dice lo siguiente:

La web de la Agencia Europea de Medicamentos (EMA) así como a otros documentos incluyen recomendaciones prácticas acerca de cómo incluir la información en las propuestas de documentos de FT, etiquetado y prospecto de las solicitudes de autorización de medicamentos y modificaciones para su 
"Ruido" en el proceso de comunicación en los prospectos de los medicamentos,
como parte de la Literatura Gris.

evaluación por la Agencia Española de Medicamentos y Productos Sanitarios (AEMPS).

De igual forma, la Directiva 2001/83/CE del Parlamento Europeo y del Consejo de 6 de noviembre de 2001 por la que se establece un código comunitario sobre medicamentos para uso humano (versión consolidada), detalla la información que debe ser incluida en la FT, etiquetado y prospecto de los medicamentos de uso humano. Esta información está, asimismo, recogida en las plantillas QRD, documentos elaborados por el grupo QRD de la EMA, donde se indica como disponer esta información de forma normalizada. La AEM también informa en su web sobre el vocabulario que se ha de incluir en los prospectos y hace la siguiente referencia al mismo:

Prospecto: El tamaño de letra empleado debe ser lo más grande posible para facilitar la lectura. Se debe considerar un tamaño mínimo de letra de 9 puntos, medido en la fuente 'Times New Roman' (not narrowed) con un espacio entre líneas de al menos $3 \mathrm{~mm}$. (Directriz de legibilidad).

La AEM incluye, también, la utilización de dibujos en la información del medicamento, la Agencia Española del Medicamento dice lo siguiente:

Únicamente se considera aceptable la inclusión de dibujos/ pictogramas/imágenes que ayuden a la comprensión de la información recogida en el etiquetado, ej., formas farmacéuticas, siempre y cuando sean un reflejo de la forma farmacéutica incluida en el envase. En ningún caso se permitirá la inclusión de pictogramas/dibujo/ imágenes de carácter promocional, esto es, que su presencia (1) suponga una clara incitación a promover la prescripción, la dispensación, la venta o el consumo de medicamentos, (2) no aporte ningún tipo de información adicional al paciente (ej. nubes, flores, etc.) y/o (3) no esté relacionado con la información contenida en la información del medicamento.

La AEM diferencia entre medicamentos sujetos, o no, a prescripción facultativa. En los medicamentos sujetos a prescripción médica no está permitida la inclusión de dibujos que aludan a las propiedades del medicamento en cuestión. En medicamentos no sujetos a prescripción médica sí se acepta la inclusión de dibujos que faciliten la comprensión por parte del paciente de la indicación terapéutica a la que va destinado el medicamento. Las propuestas serán evaluadas caso por caso.

Teniendo en cuenta todo lo dicho anteriormente, las opiniones de los usuarios de los medicamentos es necesaria para poder encontrar una posible solución al problema.

\section{OBJETIVO}

Constatar el grado de conformidad o disconformidad de los usuarios de los medicamentos con respecto al lenguaje utilizado en los mismos.

\section{MÉTODO}

El estudio sobre los problemas de "ruido" en la comunicación a través de los prospectos de los medicamentos ha sido realizado a través de una encuesta. Se ha utilizado la herramienta de Google Forms para crear la encuesta, registrar resultados y dar la posibilidad al mayor número de gente para contestar. Es una encuesta 
"Ruido" en el proceso de comunicación en los prospectos de los medicamentos, como parte de la Literatura Gris.

anónima. En esta encuesta se ha buscado, y obtenido, información sobre tres aspectos fundamentales:

- Si la información de los prospectos es adecuada para los consumidores.

- Sí esta información es fácil de entender.

- Finalmente, la búsqueda de propuestas para mejorar la comprensión del mensaje informativo de los prospectos.

\section{ANÁLISIS DE RESULTADOS}

La participación total en la encuesta ha sido de 407 personas durante un tiempo de 28 días empezando en el mes de Julio. Las respuestas difieren de una pregunta a otra, siendo la media de contestaciones de 403.

Más de la mitad de las personas que contestaron a las preguntas de la encuesta son mujeres (56\%), frente a los hombres que representan un $43 \%$. Un $1 \%$ ha preferido no contestar sobre su sexo. Ateniéndonos a estos resultados de participación la conclusión es que las mujeres están más interesadas y preocupadas por leer y entender los prospectos.

De esta manera, la participación entre las personas pertenecientes a distintos grupos de edad, queda de la siguiente manera:

- +65: $12 \%$

- 55 a $64: 29 \%$

- 45 a $54: 23 \%$

- 35 a $44: 15 \%$

- 25 a $34: 12 \%$

- $\quad 18$ a $24: 9 \%$

De acuerdo con estos datos, resulta evidente que a menor edad hay una menor preocupación por entender el mensaje de los prospectos. Esto puede entenderse ya que durante la juventud la percepción de riesgos y enfermedades es menor y, por el contrario, está aumenta según va aumentando la edad. Para los mayores de 65 años la participación se reduce y se puede deber a varias causas: el índice de mortalidad es mayor; las personas de este grupo no leen los prospectos y son médico y enfermeras o familiares quienes se ocupan de esta cuestión.

La encuesta fue programada para todos los territorios de España. La Comunidad de Madrid es la que tiene una mayor participación, seguida de Andalucía. Castilla la Mancha junto a Castilla León y Extremadura, que también alcanzan una participación considerable. En el resto de Comunidades Autónomas la participación es mucho menor. Hay que resaltar que ha habido participación en todas las Comunidades Autónomas con la excepción de la Ciudad Autónoma de Melilla donde la participación ha sido inexistente.

Ante la pregunta sobre los hábitos de lectura de los prospectos de los medicamentos los porcentajes son los siguientes: Un53\% han respondido afirmativamente diciendo que leen habitualmente los prospectos. Un $8 \%$ dicen que no leen los prospectos y el $39 \%$ ha contestado que lee los prospectos algunas veces.

Revista de Comunicación y Salud, 2018, Vol. 8, n² 2, pp. 41-49 
"Ruido" en el proceso de comunicación en los prospectos de los medicamentos, como parte de la Literatura Gris.

Sobre la importancia de la información contenida en los prospectos La gran mayoría de las personas $(74 \%)$ han respondido que si consideran importante la información que aparece en los prospectos. Sólo un $1 \%$ considera que la información no es importante, mientras que un $25 \%$ duda sobre la importancia de la información contenida en los prospectos.

Con respecto a los problemas que se tienen para entender la información que aparece en los prospectos de los medicamentos, las respuestas a esta pregunta demuestran la tesis de lo que se sospechaba a priori, la mayoría de la gente tiene dificultades, en algún momento o siempre, para poder entender la información contenida en los prospectos. Así el $77 \%$ de la población confiesa tener problemas ocasionalmente para entender los prospectos. El 9\% considera que siempre tiene problemas de comprensión y un $14 \%$ afirma no tener nunca problemas para una perfecta comprensión de la información.

¿Es realmente importante entender la información que aparece en los prospectos? El $83 \%$ de la población considera que es importante comprender la información sobre los medicamentos. Un $15 \%$ no dice nada mientras que un $2 \%$ no cree que entender bien la información de los prospectos sea verdaderamente importante.

Finalmente y ante tres opciones para mejorar la comunicación las respuestas muestran que un $71 \%$ piensa que se debe utilizar un vocabulario más simple, evitando los tecnicismos y la jerga propia de los médicos. El 16\% considera que hay que reducir la información sólo a aquello realmente importante, mientras que un 13\% cree que lo mejor es recibir una explicación previa por parte del Doctor o del farmacéutico.

Entre las sugerencias que los participantes en la encuesta han señalado la gran mayoría hace referencia a la utilización de un vocabulario más sencillo, incluso coloquial, para que todo el mundo lo pueda entender sin problemas. Otro número importante no ha dicho nada contestando, No, Nada, Ninguna... y hay otra serie de respuestas que son curiosas por distintas razones. Entre ellas se han seleccionado las siguientes respuestas:

- "No tengo problemas pues soy médico pero un lenguaje más simple sería muy oportuno".

- "La letra es muy pequeña y hay demasiada información".

- "Desde el punto de vista del farmacéutico tengo que comentar que a pesar de mis consejos, el paciente hace básicamente lo que le da la gana. Creo que en líneas generales el paciente no lee el prospecto, y que la información que viene en el mismo debería ser más clara y concisa, con un vocabulario más sencillo".

- "Mi madre es médico y me explica".

- "Cuidar el vocabulario, utilizando lo más sencillo posible".

- "Me lo tienen que leer ya que soy invidente y me lo explican. Estaría bien que hubiera la posibilidad de leerlo en braille".

Con respecto a la observación de la persona invidente, la Agencia Europea del Medicamento (EMA), dice lo siguiente en una información actualizada a 13//2018:

Revista de Comunicación y Salud, 2018, Vol. 8, n² 2, pp. 41-49 
"Ruido" en el proceso de comunicación en los prospectos de los medicamentos, como parte de la Literatura Gris.

Con el fin de garantizar el acceso a la información de las personas invidentes o con discapacidad visual, el nombre de los medicamentos deberá indicarse en los envases impresos en alfabeto Braille. Las instrucciones relativas a la información mínima a incluir en braille así como los requisitos para su incorporación efectiva y posibles exenciones están recogidas en la sección 'Braille' del documento de preguntas y respuestas sobre el Real Decreto 1345/2007.

\section{CONCLUSIONES}

La importancia de la comunicación en el ámbito de la salud no se discute, y saber manejar las implicaciones emocionales, sociales y terapéuticas de la comunicación nos permite establecer relaciones altamente satisfactorias y productivas entre la población y los profesionales sanitarios (Pascual Segovia, 2012).

Después del análisis de los datos de las encuestas realizadas, se observa una primera conclusión: Existe un problema importante de comunicación. El hecho de que se señale la utilización de la jerga médica en muchos prospectos da lugar a un mal proceso de comunicación donde el "ruido" está en el código que se utiliza. Si el código no es compartido entre emisor y receptor, no hay comunicación. En el caso de la sanidad, esto supone un problema grave para una efectiva utilización del medicamento.

Las soluciones que pueden aplicarse para solucionar este problema son fácilmente comprensibles y dependen, en su mayoría, de la voluntad de las Autoridades Sanitarias y han de aplicar una mayor claridad al mensaje que el destinatario debe recibir. Es necesario adaptar el mensaje a la gran mayoría de los receptores evitando los problemas de decodificación del mismo una vez emitido por el emisor y recibido por el receptor.

De igual manera, los ciudadanos deben aceptar que el prospecto es un elemento inherente al medicamento y que su lectura y comprensión es de vital importancia para garantizar un uso correcto del mismo y de esta forma, conseguir el éxito terapéutico.

El usuario debe prestar especial atención al modo de uso y posología y cumplir la terapia impuesta por el médico.

Las figuras de médico y farmacéutico están para solucionar las dudas y por ello hay que recomendar una mayor interacción entre médico/farmacéutico y consumidores.

Finalmente, la implementación de nuevas formas en la redacción de los prospectos puede ser cara, pero es un problema que atañe a la salud de todo el mundo. En este caso, el económico debería ser el menor de los problemas.

Las encuestas que se han llevado a cabo muestran resultados reales y dan suficientes pistas para ofrecer una solución adecuada a las quejas. Además, la implementación de soluciones fáciles y creativas hará que los problemas actuales de la comunicación escrita en los prospectos de los medicamentos, se conviertan en historia.

Revista de Comunicación y Salud, 2018, Vol. 8, n² 2, pp. 41-49 
"Ruido" en el proceso de comunicación en los prospectos de los medicamentos, como parte de la Literatura Gris.

También hay que recalcar que las encuestas fueron realizadas en el verano de 2018, sin embargo, los datos son válidos a día de hoy. No se ha hecho ningún cambio significativo en la política referida a la utilización de un determinado tipo de lenguaje en el proceso de comunicación a través de los prospectos de los medicamentos.

\section{BIBLIOGRAFÍA}

Agencia Española de Medicamentos y Productos Sanitarios - AEMPS https://www.aemps.gob.es/

Agencia Europea de Medicamentos (EMA) - EUROPA | Unión Europea. Disponible en https://europa.eu/european-union/about-eu/agencies/ema_es

Agencia Española del Medicamento. Disponible en https://www.aemps.gob.es/ (Actualizado a 20/05/2018).

Alberani, V., De Castro Pietrangeli, P., \& Mazza, A. M. (1990). The use of grey literature in health sciences: a preliminary survey. Bulletin of the Medical Library Association, 78(4), 358-363.

Artículo 8.1. del Real Decreto 2236/1993, de 17 de diciembre, por el que se regula el etiquetado y prospecto de los medicamentos de uso humano.

Cascales Martínez, M. (2012). Educación farmacéutica en la interpretación de prospectos. https://tinyurl.com/y9hrajpx

Farace, D., \& Schöpfel, J. (2017). Grey literature in library and information studies. KG Saur.

García Santiago, L. (1999). Manual básico de literatura gris. El lado oscuro de la documentación. Gijón: Trea.

GL'99 Conference Program. Fourth International Conference on Grey Literature: New Frontiers in Grey Literature.GreyNet, Grey Literature. Network Service. Washington D.C. USA, 4-5 October 1999.

Grey Literature Report Website. Disponible en www.greylit.org Twitter: @nyamgreylit

Jakobson, R. (1960). Las funciones del lenguaje. La Haya: Siglo XXI.

Jakobson, R. (1981). La lingüística y la teoría de la comunicación. Ensayos de lingüística general, 79-97.

López Gutiérrez, A. y Bordonado Bermejo, M. J. (2018). "Literatura Gris: "ruido" en el Proceso de comunicación en los prospectos de los medicamentos". La Comunicación ante el Ciudadano, (pp. 287-302). Barcelona: Gedisa. 
"Ruido" en el proceso de comunicación en los prospectos de los medicamentos, como parte de la Literatura Gris.

López Martínez, M. I. y Hernández Sánchez, E. (2004). Los prospectos de los fármacos como paradigma de comunicación inefectiva. Revista de Investigación Lingüística, $\mathrm{n}^{\circ}$ de marzo 2004.

Mercado López, S. (2003). Estructura y relación de poder en los prospectos de medicamentos vendidos en España. Estudios filológicos, 38, 93-110.

Paoli, J. A. (1983). Comunicación e información. Perspectivas teóricas. México: Trillas, UAM.

Pascual Segovia, M. J. (2012). Reseña: Comunicar y curar. Un desafío para pacientes y profesionales sanitarios. Revista de Comunicación y Salud, 13(1), 6771. https://tinyurl.com/hr5dtj7

Pérez García, E. (2004). Los prospectos: estudio de lo tecnoletal hacia lo divulgativo. Tonos. Revista electrónica de estudios filológicos, 8. Disponible en http://www.um.es/tonosdigital/znum8/corpora/3-prospectos.htm

Pfizer España (Actualizada la información el 18/01/2017). Entender el prospecto de un medicamento. Disponible en https://tinyurl.com/y9sjljya

Yerena, M. D. S. F., \& Pérez, A. C. (2016). Comunicación oral y escrita. Pearson Educación. 